01

\title{
Метод определения работы выхода электронов и позитронов из металла, содержащего вакансии
}

\author{
(C) В.В. Погосов \\ Запорожский национальный технический университет, \\ Запорожье, Украина \\ E-mail: vpogosov@zntu.edu.ua \\ (Поступила в Редакцию 10 октября 2016 г.)
}

Предлагается метод, в котором комбинируются самосогласованные решения для моновакансии в металле без учета внешней поверхности и решение в модели стабильного желе для металла с однородным объемом и плоской поверхностью, но пониженной плотностью вследствие наличия сверхрешетки пустот-вакансий относительной концентрации $c_{v}$. При использовании $c_{v}$ в качестве малого параметра все энергетические характеристики раскладываются в функциональный ряд. Нулевые члены разложения относятся к бездефектному металлу, а линейные по $c_{v}$ поправки выражены через его характеристики. Получены точные формулы, позволяющие учесть влияние вакансий на работу выхода электронов и позитронов. Вычисления характеристик при различных температурах для Al и Na проведены методом Кона-Шема. Дан краткий очерк применения метода для сферических кластеров.

DOI: 10.21883/FTT.2017.06.44475.374

1. Теория плавления Френкеля предполагает скачкообразное увеличение концентрации вакансий в тройной точке, а также уменьшение энергии образования вакансий с ростом их концентрации [1]. Равновесная концентрация вакансий вычисляется из термодинамических соображений при наличии энергии образования вакансии, величина которой может быть извлечена из данных позитронной аннигиляционной спектроскопии. В точке плавления относительная концентрация вакансий в металлах составляет доли процента. Несмотря на столь малые концентрации, вакансии оказывают большое влияние на свойства твердых тел. В случае радиационных повреждений в металлах концентрация вакансий может составлять десятки процентов.

Экспериментально установлено, что температура плавления свободных кластеров уменьшается с уменьшением их размеров. Этот факт стимулирует повышенный интерес к рассмотрению фазового перехода из твердого в жидкое состояние как конфигурационного возбуждения пустот-вакансий в кластерах [2].

Трансляционная симметрия решетки твердого тела лежит в основе расчета работы выхода. Поэтому чтобы учесть влияние вакансий на работу выхода, приходится предполагать их периодическое расположение в виде „сверхрешетки“ в металле (см. рисунок). Только в этом случае можно говорить о вакансионном сдвиге дна зон электронной и позитронной проводимости.

Сложность заключается в том, что объем металла вследствие наличия вакансий является неоднородным, поэтому методом функционала плотности в модели желе потребуется решать трехмерную задачу. В одномерной задаче невозможно одновременно описать набор сферически-симметричных вакансий и плоскую внешнюю поверхность металла.

Можно предложить подход, в котором комбинируются решения для моновакансии в металле без учета внешней поверхности и решение для металла с однородным объемом, но пониженной плотностью вследствие наличия сверхрешетки вакансий. В этом случае эффективная работа выхода электронов $W_{\mathrm{eff}}^{e}$ и позитронов $W_{\mathrm{eff}}^{p}$ из металла может быть представлена в виде суммы

$$
W_{\mathrm{eff}}^{e, p}=W^{e, p}+\delta W_{v}^{e, p},
$$

где $W^{e, p}$ - традиционно рассчитываемая методом функционала плотности характеристика, состоящая из объемной компоненты и поверхностного дипольного барьера.

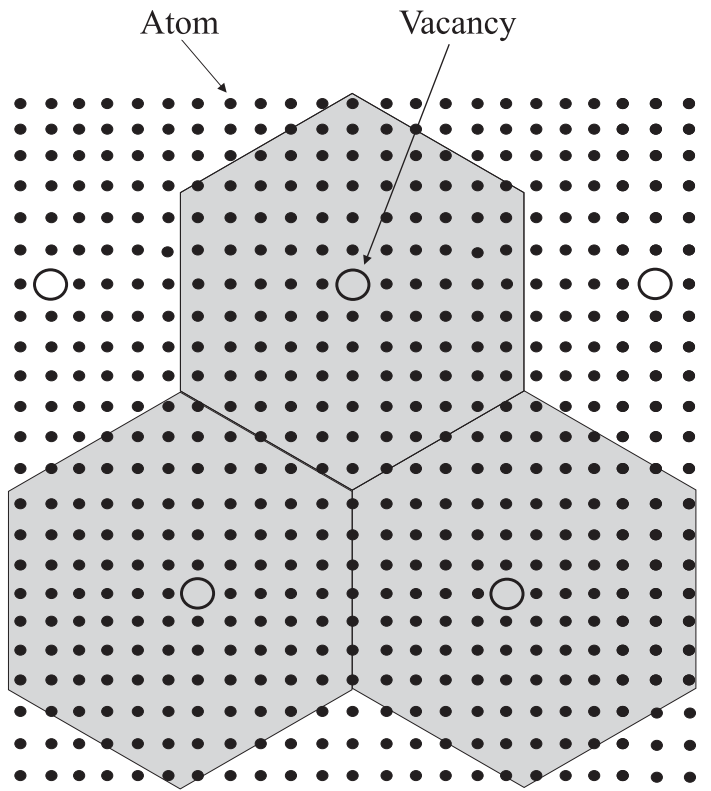

Иллюстрация сверхрешетки вакансий $\left(c_{v} \sim 10^{-3}\right)$. В модели элементарная ячейка-многогранник вакансионной сверхрешетки заменяется на сферическую ячейку того же объема. 
В рамках метода Кона-Шема и модели стабильного желе в работах [3,4] самосогласованно решена задача о рассеянии электронов и позитронов на моновакансии в металлах. Вычисленные фазы рассеяния и представление системы вакансий как „сверхрешетки“ в металле позволили найти сдвиг энергии основного состояния электронов и позитронов в объеме металла. Величина $\delta W_{v}^{e, p}$ в (1) - это вакансионный вклад в объемную компоненту работы выхода.

Целью настоящей работы является разработка метода определения вакансионной зависимости работы выхода электронов и позитронов методом функционала плотности.

2. Запишем большой термодинамический потенциал $\Omega[n]$ системы металл-вакуум с давлением $P$ и объемом $V$ в виде функционала неоднородной электронной концентрации $n(r)$

$$
\begin{aligned}
\Omega[n]= & G[n]-\mu \int d \mathbf{r} n(\mathbf{r})+\frac{e}{2} \int d \mathbf{r} \phi(\mathbf{r})[n(\mathbf{r}-\rho \mathbf{r})] \\
& -\Delta \bar{\varepsilon} \int d \mathbf{r} \rho(\mathbf{r})+\langle\delta v\rangle_{\mathrm{WS}} \\
& \times \int d \mathbf{r} \theta(\mathbf{r}-\mathbf{R})[n(\mathbf{r})-\rho(\mathbf{r})]=P V .
\end{aligned}
$$

Первое слагаемое $(2)-G[n]-$ представляет собой универсальный функционал, соответствующий модели обычного желе с энергией $\varepsilon_{J}=\varepsilon_{t}+\varepsilon_{\mathrm{xc}}\left(\varepsilon_{t}\right.$ и $\varepsilon_{\mathrm{xc}}-$ кинетическая и обменно-корреляционная энергия электронного газа на один электрон), приходящейся на один электрон; $\mu-$ химический потенциал электронов; третье слагаемое учитывает электростатическое взаимодействие в неоднородном металле $(e-$ единичный положительный заряд); последние два члена учитывают структуру ионной подсистемы [5]. Распределение положительного заряда $\rho(\mathbf{r})$ задается однородным внутри металла и равным нулю вне его. Координата $\mathbf{R}$ соответствует границе металл-вакуум; $\theta-$ единичная ступенчатая функция.

Обозначим чертой сверху значения величин в объеме однородного металла. Энергия на один электрон в объеме состоит из компоненты обычного желе, энергии Маделунга и псевдопотенциальной поправки

$$
\begin{gathered}
\bar{\varepsilon}=\bar{\varepsilon}_{J}+\Delta \bar{\varepsilon}, \quad \Delta \bar{\varepsilon}=\varepsilon_{M}(\bar{n})+\bar{w}_{R}\left(\bar{n}, r_{c}\right) \\
\langle\delta v\rangle_{\mathrm{WS}}=\bar{n} \frac{\partial \Delta \bar{\varepsilon}}{\partial \bar{n}}=\tilde{\varepsilon}+\varepsilon_{M}+\bar{w}_{R}, \quad \bar{w}_{R}=2 \pi e^{2} \bar{n} r_{c}^{2} \\
\varepsilon_{M}(\bar{n})=-\frac{9 Z e^{2}}{10 r_{0}}, \quad \tilde{\varepsilon}=-\frac{2}{3} \varepsilon_{M}
\end{gathered}
$$

где $\bar{w}_{R}-$ усредненная по ячейке Вигнера-Зейтца (радиуса $r_{0}=Z^{1 / 3} r_{s}$ ) разница между псевдопотенциалом иона и электростатическим потенциалом однородного положительно заряженного фона, $r_{c}$ - радиус псевдопотенциала Ашкрофта, $\varepsilon_{M}(\bar{n})$ - энергия Маделунга точечных ионов с валентностью $Z$, погруженных в однородный отрицательно заряженный фон, $\tilde{\varepsilon}-$ собственная электростатическая энергия однородного отрицательного фона внутри ячейки. Концентрация электронов удовлетворяет условию $4 / 3 \pi r_{s}^{3} \bar{n}=1$.

Вследствие механического равновесия металла с вакуумом давление в его объеме $P=-\bar{n}^{2}(\partial \bar{\varepsilon} / \partial \bar{n})=0$. Отсюда следуют соотношения

$$
\frac{d \bar{\varepsilon}_{J}}{d \bar{n}}=-\frac{\partial \Delta \bar{\varepsilon}}{\partial \bar{n}}, \quad\langle\delta v\rangle_{\mathrm{WS}}=-\bar{n} \frac{d \bar{\varepsilon}_{J}}{d \bar{n}} .
$$

Из первого соотношения в (4) находится равновесное значение $r_{c}(\bar{n})[5]$.

Для полубесконечного металла (ось $x$ перпендикулярна поверхности раздела металл $(x \leq 0)$-вакуум $(x>0))$ равновесное распределение $n(x)$ удовлетворяет уравнению Эйлера

$$
\mu(x)=e \phi(x)+\langle\delta v\rangle_{\mathrm{WS}} \theta(-x)+\frac{\delta G}{\delta n(x)}=\mathrm{const},
$$

а электростатический потенциал $\phi(x)$ - уравнению Пуассона

$$
\begin{gathered}
\frac{d^{2} \phi}{d x^{2}}=-4 \pi e[n(x)-\rho(x)], \\
\rho(x)=\bar{n} \theta(-x), \quad \phi(+\infty)=0 .
\end{gathered}
$$

Из условия $\mu(x)=$ const следует равнозначность выбора координаты в (5). Удобно принять $x \rightarrow-\infty$, когда градиентные члены стремятся к нулю. Тогда работа выхода электронов может быть найдена как

$$
W^{e}=-\mu(-\infty) \equiv-\bar{\mu} .
$$

Сравним случаи сплошного металла и металла, содержащего вакансии. Механизм образования вакансий здесь не важен. Это может быть механизм Шоттки или механизм „выдувания“ вакансий, предложенный в [6]. Важно, что число атомов в образце настолько велико, что в межвакансионном объеме плотность вещества такая же, как и в сплошном образце.

Проведем усреднение концентраций зарядов по вакансионной ячейке сверхрешетки (см. рисунок). В результате этого условие электронейтральности в объеме „нового“ (бездефектного) металла имеет вид

$$
\bar{n}=\bar{\rho}=\frac{Z \bar{n}_{a}}{1+c_{v}},
$$

где $n_{a}$ - концентрация атомов, $c_{v}-$ относительная концентрация вакансий (безразмерный малый параметр).

Проводя разложение в ряд по малым $c_{v}$ в условии (8) и ограничиваясь линейными по $c_{v}$ слагаемыми, запишем

$$
\bar{n}=\bar{n}^{0}+\bar{n}^{1} c_{v}, \quad \bar{n}^{1}=-\bar{n}^{0},
$$

где $\bar{n}^{0}-$ концентрация электронов сплошного металла $\left(c_{v}=0\right)$. 
Величины (в $\mathrm{eV})$, выделенные с помощью расчетов методом Кона-Шема для бездефектного металла в модели стабильного желе [7], вакансионные вклады в работу выхода электронов $W^{1, e}(16)$ и позитронов $W^{1, p}(18)$, а также $W_{\mathrm{eff}}^{1, e, p}(19)$, рассчитанные для различных температур $\left(a_{0}-\right.$ боровский радиус)

\begin{tabular}{c|c|c|c|c|c|c|c|c|c|c}
\hline Металл & $r_{s}^{0}, a_{0}$ & $T, \mathrm{~K}$ & $-e \bar{n}^{0}\left(\bar{\phi}^{0}\right)^{\prime}$ & $B^{0} / \bar{n}^{0}$ & $\bar{n}^{0}\left(\bar{\varepsilon}_{J}^{0}\right)^{\prime}$ & $-W^{1, e} / c_{v}$ & $-W_{\mathrm{eff}}^{1, e} / c_{v}$ & $-\bar{n}^{0}\left(\bar{\varepsilon}_{\mathrm{cor}}^{0}\right)^{\prime}$ & $-W^{1, p} / c_{v}$ & $-W_{\mathrm{eff}}^{1, p} / c_{v}$ \\
\hline \multirow{2}{*}{$\mathrm{Al}$} & 2.055 & 0 & 3.0353 & 5.6588 & 2.5421 & 5.1656 & & 1.4490 & 1.5863 & \\
& 2.070 & 300 & 2.9907 & 5.4195 & 2.4882 & 4.9172 & 17.8 & 1.4404 & 1.5503 & 7.05 \\
& 2.078 & 466 & 2.9678 & 5.2893 & 2.4602 & 4.7817 & & 1.4359 & 1.5319 \\
& 2.101 & $933^{*}$ & 2.9026 & 4.9476 & 2.3814 & 4.4264 & & 1.4230 & 1.4796 & \\
$\mathrm{Na}$ & 3.906 & 0 & 0.8281 & 2.0227 & 0.0925 & 1.2871 & & 0.7494 & 0.0787 & \\
& 3.959 & 186 & 0.8056 & 1.8636 & 0.0723 & 1.1303 & 5.23 & 0.7351 & 0.0705 & 0.08 \\
& 3.990 & 293 & 0.7725 & 1.7725 & 0.0611 & 1.0682 & & 0.7267 & 0.0458 & \\
& 4.012 & $371^{*}$ & 0.7838 & 1.7201 & 0.0532 & 0.9895 & & 0.7209 & 0.0629 &
\end{tabular}

* Температура плавления $T_{m}$.

По аналогии с работами [7], в которых развит метод определения размерных поправок энергетических характеристик сферических кластеров радиуса $R$ в модели стабильного желе $(1 / R$ - размерный малый параметр), представим характеристики металла, содержащего вакансии, в виде

$$
\begin{gathered}
n(x)=n^{0}(x)+n^{1}(x) c_{v}+\ldots, \\
\rho(x)=\rho^{0}(x)+\rho^{1}(x) c_{v}+\ldots, \\
\phi(x)=\phi^{0}(x)+\phi^{1}(x) c_{v}+\ldots, \\
\mu(x)=\mu^{0}(x)+\mu^{1}(x) c_{v}+\ldots .
\end{gathered}
$$

Это позволяет разложить уравнения (5) и (6) в функциональный ряд. Ограничившись нулевым и первым порядком разложения (верхние индексы 0 и 1 соответственно), имеем

$\mu^{0}(x)=e \phi^{0}(x)+\frac{\partial g^{0}}{\partial n^{0}}+\langle\delta v\rangle_{\mathrm{WS}}^{0} \theta(-x)+$ gradient terms

$$
\begin{aligned}
\mu^{1}(x)= & e \phi^{1}(x)+n^{1}(x) \frac{\partial^{2} g^{0}}{\partial\left(n^{0}\right)^{2}} \\
& +\bar{n}^{1} \frac{\partial\langle\delta v\rangle_{\mathrm{WS}}^{0}}{\partial \bar{n}^{0}} \theta(-x)+\text { gradient terms, }
\end{aligned}
$$

$\frac{d^{2} \phi^{0,1}(x)}{d x^{2}}=-4 \pi e\left[n^{0,1}(x)-\rho^{0,1}(x)\right], \quad \phi^{0,1}(+\infty)=0$.

В (11) $g^{0} \equiv n^{0}(x) \varepsilon\left(n^{0}(x)\right)$ - объемная плотность энергии в приближении локальной плотности.

При $x=-\infty$ получаем

$$
\begin{gathered}
\bar{\mu}^{0}=e \bar{\phi}^{0}(x)+\frac{\partial \bar{g}^{0}}{\partial \bar{n}^{0}}+\langle\delta v\rangle_{\mathrm{WS}}^{0}=-W^{0, e}, \\
\bar{\mu}^{1}=e \bar{\phi}^{1}+\bar{n}^{1} \frac{\partial^{2} \bar{g}^{0}}{\partial\left(\bar{n}^{0}\right)^{2}}+\bar{n}^{1} \frac{\partial\langle\delta v\rangle_{\mathrm{WS}}^{0}}{\partial \bar{n}^{0}}=e \bar{\phi}^{1} \\
+\bar{n}^{1}\left[\bar{n}^{0}\left(\bar{\varepsilon}_{S J}^{0}\right)^{\prime \prime}+\left(\bar{\varepsilon}_{J}^{0}\right)^{\prime}\right]=-W^{1, e} / c_{v} .
\end{gathered}
$$

Интегрирование в (12) дает

$$
\bar{\phi}^{0,1}=-4 \pi e \int_{-\infty}^{\infty} d x x\left[n^{0,1}(x)-\rho^{0,1}(x)\right] .
$$

Вследствие того что вакансионный сдвиг электростатического потенциала в объеме $\bar{\phi}^{1}$ обусловлен только уменьшением в среднем концентрации электронов и ионов в металле по аналогии с самосжатыми кластерами [7], где эффект обратный, вместо (14) запишем

$$
\bar{\phi}^{1}=\bar{n}^{1}\left(\bar{\phi}^{0}\right)^{\prime} .
$$

Здесь и далее „штрих“ означает производную по $\bar{n}^{0}$.

Используя выражение для объемного модуля сжатия $B^{0}=\left(\bar{n}^{0}\right)^{3}\left(\bar{\varepsilon}^{0}\right)^{\prime \prime}$, а также формулы (9) и (15), окончательно получим для $W^{e}=W^{0, e}+W^{1, e}$

$$
W^{1, e}=-\left[e \bar{n}^{0}\left(\bar{\phi}^{0}\right)^{\prime}+\left(\frac{B^{0}}{\bar{n}^{0}}+\bar{n}^{0}\left(\bar{\varepsilon}_{J}^{0}\right)^{\prime}\right)\right] c_{v} .
$$

В результате такого подхода вакансионный вклад в работу выхода электрона выражается только через характеристики бездефектного металла.

По аналогии можно записать выражение для работы выхода позитрона из сплошного металла

$$
W^{0, p}=e \bar{\phi}^{0}-\bar{\varepsilon}_{\mathrm{cor}}^{0}-E_{0},
$$

где первое слагаемое соответствует электростатическому взаимодействию позитрона с металлом, второе представляет вклад позитрон-электронных корреляций в приближении локальной плотности [8], третий член описывает неэлектростатическое позитрон-ионное взаимодействие в каждой из атомных ячеек Вигнера-Зейтца. Вакансионная поправка к работе выхода позитрона также выражается через характеристики металла в отсутствие вакансий

$$
W^{1, p}=\left[e \bar{n}^{0}\left(\bar{\phi}^{0}\right)^{\prime}-\bar{n}^{0}\left(\bar{\varepsilon}_{\text {cor }}^{0}\right)^{\prime}\right] c_{v} .
$$

3. В приведенных выше точных формулах единственные величины, которые требуют самосогласованных вычислений, - значение электростатического потенциала 
в объеме вдали от геометрической границы металла $\bar{\phi}^{0}$ и его производная $\left(\bar{\phi}^{0}\right)^{\prime}$. В таблице приведены значения компонент формул в зависимости от температуры. Видно, что вклад от поверхностного барьера $\sim\left(\bar{\phi}^{0}\right)^{\prime}$ очень значителен и с разным знаком входит в $W^{1, e}$ и $W^{1, p}$, конкурируя в (16) с объемным вкладом (сумма слагаемых в круглых скобках). При нагреве металлов в твердом состоянии изменение рассчитанных величин на одну вакансию составляет проценты.

Для бездефектных $\mathrm{Al}$ и $\mathrm{Na}$ соответственно $W^{0, e}=4.30 \mathrm{eV}, \quad W^{0, p}=-0.59 \mathrm{eV} \quad$ и $\quad W^{0, e}=2.93 \mathrm{eV}$, $W^{0, p}=3.61 \mathrm{eV}$. Отрицательный знак в $W^{0, p}$ означает, что металл $(\mathrm{Al})$ для позитрона представляет собой потенциальный барьер по отношению к вакууму. Полученная в результате расчета отрицательная по знаку величина работы выхода указывает на то, что фракция свободных позитронов (не локализованных в вакансиях) способна покинуть Al в результате обратной эмиссии за время, меньшее времени жизни в металле.

Далее в соответствии с (1) запишем

$$
\begin{gathered}
W_{\mathrm{eff}}^{0, e, p}=W^{0, e, p}, \\
W_{\mathrm{eff}}^{1, e, p}=W^{1, e, p}+\delta W_{v}^{e, p},
\end{gathered}
$$

где

$$
\delta W_{v}^{e, p}=-A^{e, p} c_{v} .
$$

На основе численного решения задачи о рассеянии электронов и позитронов на вакансионном потенциале $[3,4]$ получены значения $A^{e}=12.9 \mathrm{eV}, A^{p}=5.5 \mathrm{eV}$ для $\mathrm{Al}\left(r_{s}^{0}=2.07 a_{0}\right)$ и $A^{e}=4.10 \mathrm{eV}, A^{p}=0.0095 \mathrm{eV}$ для $\mathrm{Na}\left(r_{s}^{0}=3.93 a_{0}\right)$. Вакансия для электрона в объеме представляет собой потенциальный бугор, а для позитрона - яму, в которой он может и локализоваться. В случае $\mathrm{Na}$ эта яма настолько мелкая, что локализация невыгодна. В целом величины $W^{1, e, p}$ и $W_{\mathrm{eff}}^{1, e, p}$ отрицательны. Почти во всех случаях львиную долю эффекта составляет величина $\delta W_{v}$ (см. таблицу).

Используя величину энергии образования вакансии $\varepsilon_{v}=0.66 \mathrm{eV}[9]$ и термодинамическое определение относительной равновесной концентрации вакансий, для $\mathrm{Al}$ в точке плавления мы получили значение $c_{v}\left(T_{m}\right) \approx 10^{-3}[10]$. Следовательно, вклад равновесных объемных вакансий в работу выхода электронов составляет примерно $-0.018 \mathrm{eV}$, а для позитронов $-0.007 \mathrm{eV}$. По данным работы [11] величина концентрации должна быть значительно выше, тогда соответственно и эффект от вакансий увеличится во много раз.

Для позитронов влияние вакансий выглядит незначительным. Однако в силу специфики обратной эмиссии свободные позитроны могут рассеиваться не только на пустых вакансиях, но и на вакансиях, содержащих локализованные позитроны. Расчет в [12] показывает, что такие заряженные вакансии вблизи реальной поверхности, где концентрация вакансий повышена, а сами они организованы в двумерную сверхрешетку, создают приповерхностный барьер $(\sim 1 \mathrm{eV})$, что делает невозможным участие низкоэнергетических позитронов в обратной эмиссии.

4. Кратко рассмотрим применение метода для сферического кластера радиуса $R$ с началом координат в центре $(r=0)$. Распределение вакансий зададим сферически-симметричным и равномерным по координационным слоям. Таким образом, в задаче появляются два независимых малых параметра: $1 / R$ и $c_{v}$. В квазиклассическом пределе при пренебрежении дискретностью спектра находится величина $\delta W_{v}^{e} \sim c_{v}$. Вклад от самосжатия в $\delta W_{v}^{e}$ положительный и имеет порядок $1 / R$ и выше.

По аналогии с методикой работ [7], раскладывая в ряд по малым $1 / R$, получаем серию уравнений, например, для $\mu_{0}(r)+\mu_{1}(r) / R+\ldots$. Переходим к координатам $x=r-R$, берем предел $R, N \rightarrow \infty$., поддерживая условие $c_{v}=$ const. Координата $x=0$ соответствует местоположению плоской поверхности. Далее, раскладывая полученные характеристики в другой ряд (по степеням $c_{v}$ ), получаем для электронов в линейных приближениях

$$
\bar{\mu}\left(R, c_{v}\right)=\bar{\mu}_{0}+\frac{\bar{\mu}_{1}}{R}+\bar{\mu}^{1} c_{v}+O\left(\frac{1}{R^{2}}, c_{v}^{2}, \frac{c_{v}}{R^{2}}\right) .
$$

Заметим, что $\bar{\mu}_{0}$ и $\bar{\mu}^{0}$ совпадают, а $\bar{\mu}_{1}$ и $\bar{\mu}^{1}$ имеют разные размерности и выражаются через характеристики бездефектного металла с плоской поверхностью. Окончательным результатом будет асимптотическое выражение для потенциала ионизации кластера

$$
\begin{aligned}
\mathrm{IP}= & -\bar{\mu}_{0}-\frac{\bar{\mu}_{1}}{R}-\bar{\mu}^{1} c_{v}+\delta W_{v}^{e}\left(R, c_{v}\right) \\
& +\frac{e^{2}}{2 C}\left(1-\frac{1}{3} c_{v}\right)+O\left(\frac{1}{R^{2}}, c_{v}^{2}, \frac{c_{v}}{R^{2}}\right),
\end{aligned}
$$

где $C=R$ - электрическая емкость сплошного кластера. Число атомов в сплошном и дефектном кластерах одно и то же. Выражение (22) в пределе $R \rightarrow \infty$ переходит в формулы (19).

5. В заключение отметим, что полученные результаты для работы выхода полубесконечного металла справедливы в линейном по $c_{v}$ приближении, а также в предположении равенства нулю давления в металле. В поправки $\sim c_{v}^{2}$ уже войдут производные $d A^{e, p} / d \bar{n}_{0}$ (см. (20)). Для их вычисления необходимо использовать методику работ $[3,4]$.

\section{Список литературы}

[1] Я.И. Френкель. Кинетическая теория жидкостей. Изд-во АН СССР, М.-Л. (1959). 460 c.

[2] R.S. Berry, B.M. Smirnov. ЖЭТФ 125, 414 (2004); R.S. Berry, B.M. Smirnov. Phys. Rep. 527, 205 (2013).

[3] А.В. Бабич, П.В. Вакула, В.В. Погосов. ФТТ 56, 841 (2014).

[4] А.В. Бабич, П.В. Вакула, В.В. Погосов. ФТТ 56, 1671 (2014).

[5] J.P. Perdew, H.Q. Tran, E.D. Smith. Phys. Rev. B 42, 11627 (1990). 
[6] V.V. Pogosov. Solid State Commun. 89, 1017 (1994).

[7] I.T. Iakubov, V.V. Pogosov. Physica A 214, 287 (1995); A. Kiejna, V.V. Pogosov. J. Phys.: Condens. Matter 8, 4245 (1996).

[8] N.D. Drummond, P. Lopez Rios, R.J. Needs, C.J. Pickard. Phys. Rev. Lett. 107, 207402 (2011).

[9] Y. Kraftmakher. Phys. Rep. 299, 79 (1998); C. Freysoldt, B. Grabowski, T. Hickel, J. Neugebauer, G. Kresse, A. Janotti, C.G. Van de Walle. Rev. Mod. Phys. 86, 253 (2014).

[10] М.В. Штремель. Прочность сплавов. Дефекты решетки. Металлургия, М. (1982). 278 с.

[11] E.M. Gullikson, A.P. Mills, jr. Phys. Rev. B 35, 8759 (1987).

[12] А.В. Бабич, В.В. Погосов, В.И. Рева. ФТТ 57, 2081 (2015). 